\title{
Negative polarity items: a case for questions as licensers*
}

\author{
Bernhard Schwarz \\ McGill University
}

\begin{abstract}
Existing analyses of NPI licensing in questions instantiate two different approaches. One approach holds that questions are NPI licensers in their own right (Kadmon \& Landman 1990; Krifka 1995, 2003; van Rooy 2003); the other holds that, in virtue of their syntax, questions host silent expressions that do the licensing for them, such as a silent version of exclusive only (Nicolae 2013, 2015) or negation not (Guerzoni \& Sharvit 2014). Based one a pattern of NPI licensing in alternative questions, this paper presents a case for the former approach. Specifically, it offers an argument for the analysis developed in Krifka 1995, 2003 and van Rooy 2003, which centrally refers to questions' information theoretic entropy (Shannon 1948).
\end{abstract}

Keywords: questions, negative polarity items, entropy, uniqueness presupposition

\section{Introduction}

Weak negative polarity items (NPIs) like unstressed existential any or ever are known to be licensed in information seeking questions, including polar questions and wh-questions (e.g., Krifka 1995).

a. Did Ann eat anything?

b. Which of the children ate anything?

Two types of analyses have emerged. The questions-are-licensers (QAL) approach holds that questions are NPI licensers in their own right, in the sense that the theory of NPI licensing refers to questions (Kadmon \& Landman 1990; Krifka 1995, 2003; van Rooy 2003). The questions-introduce-licensers (QIL) approach, in contrast, denies that the theory of NPI licensing refers to questions; it holds instead that in virtue of their syntax, questions can host silent expressions that do the licensing for them, such as a silent version of exclusive only (Nicolae 2013, 2015) or negation not

* For discussion related to this project, thanks to Luis Alonso-Ovalle, Dan Goodhue, Tim O'Donnell, Junko Shimoyama, and Michael Wagner, as well as the (other) members of the McGill Semantics Research Group. Thanks to Brian Buccola, Dan Goodhue, Emily Kellison-Linn, and Vincent Rouillard for providing their English judgments. This research was supported by the Social Sciences and Humanities Research Council (SSHRC), grants \#435-2016-1448 and \#435-2013-0592. 
Questions as licensers

(Guerzoni \& Sharvit 2014), which are known to license NPIs outside the question context as well.

Most recently, the QIL approach has been the more conspicuous in the literature (Nicolae 2013; Guerzoni \& Sharvit 2014; Nicolae 2015). Here I will offer a case for the QAL approach. Specifically, I will put forward an argument for an analysis developed in Krifka 1995 and van Rooy 2003, which centrally employs the information-theoretic notion of entropy (Shannon 1948; Cover \& Thomas 2012).

I do so by identifying a benefit of that account that so far appears to have gone unnoticed: it solves a puzzle regarding the licensing of weak NPIs in socalled alternative questions, which here I will refer to as disjunctive questions. Disjunctive questions can be classified as polar or phrasal, as illustrated in (2). ${ }^{1}$ The generalization suggested by the contrast in (3), the puzzle I will set out to solve, is that polar, but not phrasal, disjunctive questions license weak NPIs. ${ }^{2}$

a. Did Ann eat something or not?

polar

b. Did ANN eat something or BEN?

phrasal

a. Did Ann eat anything or not?

polar

b. \#Did ANN eat anything or BEN?

phrasal

To preview, under the QAL analysis to be explored here, NPI licensing in questions is dependent on syntactic form only in so far as the latter determines semantic interpretation. Assuming that a polar disjunctive question shares the semantics of its (non-disjunctive) polar counterpart (e.g., Guerzoni \& Sharvit 2014), it is immediately predicted that the two behave alike with regard to NPI licensing. As for the central case of phrasal disjunctive questions, it is their presupposition of existence and uniqueness (e.g., Biezma \& Rawlins 2012) that will be seen to interfere with NPI licensing in virtue of its effects on information theoretic question entropy.

1 Phrasal disjunctive questions have a prosodic signature that typically includes focus prosody on the parallel elements in the two disjuncts, such as Ann and Ben in (2b), where focus prosody is marked by capitalization. For details, see Biezma \& Rawlins 2015 and references cited there.

2 This generalization seems quite robust. In particular, there was no disagreement about (3a) or (3b) among the four English speakers I consulted. In assuming the generalization to be valid, I set aside Guerzoni \& Sharvit's (2014) report that polar disjunctive questions do not license weak NPIs. (The judgment Guerzoni \& Sharvit report is also in conflict with Bolinger 1978, where the acceptability of Have you ever been there or not? is taken for granted.) I likewise set aside Nicolae's (2013) report that NPIs are licensed in phrasal disjunctive questions as long as they meet certain syntactic conditions. Disagreeing with Nicolae's portrayal of the data, and consistent with judgments provided by my English speaker consultants, Guerzoni \& Sharvit (2014) (like Ladusaw 1979 and Higginbotham 1993) suggest that phrasal disjunctive questions fail to license NPIs across the board. 


\section{A baseline theory}

As a point of reference, (4) outlines a baseline theory of weak NPI licensing, broadly aligned with Kadmon \& Landman 1993, as developed in Krifka 1995, Lahiri 1998, and Chierchia 2013.

$$
\begin{array}{lll}
\text { i. } & \forall \mathrm{f}[\mathrm{f} \in|\mathrm{NPI}| \rightarrow \mathrm{f} \subset\|\mathrm{NPI}\|] & \text { NPI semantics } \\
\text { ii. } & \forall \mathrm{p}[\mathrm{p} \in|\ldots \mathrm{NPI} \ldots| \rightarrow\|\ldots \mathrm{NPI} \ldots\| \subset \mathrm{p}] & \text { NPI condition }
\end{array}
$$

The baseline theory assumes that, in addition to the actual denotation $\|\phi\|$, grammar assigns a linguistic expression $\phi$ a set of alternative semantic values $|\phi|$. According to clause (i), the NPI semantics, the actual denotation of a NPI is strictly weaker than any of the alternatives; clause (ii), the NPI condition, requires that for some larger syntactic domain [...NPI ...], this strength relation be reversed, with the domain's actual denotation being strictly stronger than each of the alternatives.

Instantiating the NPI semantics for the case of existential anything, as shown in (5), grammar is taken to determine an alternative set whose members are quantifiers that differ from anything's actual denotation in that they have a narrower domain (Kadmon \& Landman 1993; Krifka 1995; Chierchia 2013).

$$
\begin{aligned}
& \text { a. }|| \text { anything } \|=\lambda P \cdot \lambda w . \exists x \in D[P(x)(w)] \\
& \text { b. } \mid \text { anything } \mid=\left\{\lambda P . \lambda w . \exists x \in D^{\prime}[P(x)(w)]: D^{\prime} \subset D\right\}
\end{aligned}
$$

Alternatives are assumed to expand through point-wise composition (Hamblin 1973; Rooth 1985), yielding sets of alternative properties for a verb phrase like ate anything in (6), and alternative propositions for clauses like Ann ate anything in (7) or Ann did not eat anything in (8).

a. $\|$ ate anything $\|=\lambda \mathrm{y} \cdot \lambda \mathrm{w} . \exists \mathrm{x} \in \mathrm{D}[\mathrm{y}$ ate $\mathrm{x}$ in $\mathrm{w}]$

b. $\quad \mid$ ate anything $\mid=\left\{\lambda \mathrm{y} \cdot \lambda \mathrm{w} . \exists \mathrm{x} \in \mathrm{D}^{\prime}[\mathrm{y}\right.$ ate $\mathrm{x}$ in $\left.\mathrm{w}]: \mathrm{D}^{\prime} \subset \mathrm{D}\right\}$

a. $\quad \|$ Ann ate anything $\|=\lambda \mathrm{w} . \exists \mathrm{x} \in \mathrm{D}$ [Ann ate $\mathrm{x}$ in $\mathrm{w}]$

b. $\mid$ Ann ate anything $\mid=\left\{\lambda \mathrm{w} . \exists \mathrm{x} \in \mathrm{D}^{\prime}[\right.$ Ann ate $\mathrm{x}$ in $\left.\mathrm{w}]: \mathrm{D}^{\prime} \subset \mathrm{D}\right\}$

a. $\|$ not [Ann ate anything] $\|=\lambda \mathrm{w} . \neg \exists \mathrm{x} \in \mathrm{D}$ [Ann ate $\mathrm{x}$ in $\mathrm{w}$ ]

b. $\mid \operatorname{not}\left[\right.$ Ann ate anything] $\mid=\left\{\lambda \mathrm{w} . \neg \exists \mathrm{x} \in \mathrm{D}^{\prime}[\right.$ Ann ate $\mathrm{x}$ in $\left.\mathrm{w}]: \mathrm{D}^{\prime} \subset \mathrm{D}\right\}$

As intended (Ladusaw 1979), it follows that the NPI condition in (4) is not met unless anything is interpreted in the scope of an entailment reversing operator, such as negation not. So, (9a) is false but (9b) is true, capturing the basic contrast between (10a) and (10b).
a. $\forall \mathrm{p}[\mathrm{p} \in \mid$ Ann ate anything $\mid \rightarrow \|$ Ann ate anything $\| \subset \mathrm{p}]$
b. $\forall \mathrm{p}[\mathrm{p} \in \mid$ not $[$ Ann eat anything] $\mid \rightarrow \| \operatorname{not}[$ Ann ate anything] $\| \subset \mathrm{p}]$ 
Questions as licensers

a. *Ann ate anything.

b. Ann didn't eat anything.

Before moving on, let me point out that, as long as the domain D, and hence the alternative set for anything in (5b), is non-empty, it includes the impossible quantifier, the quantifier that maps any input property to the contradictory proposition. Given that the quantificational force of anything is existential, this is guaranteed by that fact that any non-empty domain D has the empty set as a proper subset. Accordingly, if non-empty, the alternative set for ate anything in (6b), will include the impossible property, the property of individuals that maps any input individual to the contradictory proposition; and the alternative sets in (7b) and (8b) will include the contradictory and tautological proposition, respectively. The existence of such trivial alternatives will become relevant in section 5 .

\section{Questions as licensers}

The QAL approach extends a baseline theory like (4) so as to render it applicable in cases where the relevant syntactic domain $[\ldots N P I \ldots]$ referred to in the NPI condition is a question. The baseline theory requires an extension because the (strict) entailment relation $\subset$ that (4) appeals to is not applicable to questions in the first place. Kadmon \& Landman (1990), Krifka (1995, 2003), and van Rooy (2003) accordingly search for a suitable ordering relation $>$ that applies to questions in the right way, to be substituted for the strict entailment relation $\subset$. With such an ordering relation $>$ given, an NPI theory for questions takes the form in (11).

$$
\begin{array}{lll}
\text { i. } & \forall \mathrm{f}[\mathrm{f} \in|\mathrm{NPI}| \rightarrow \mathrm{f} \subset\|\mathrm{NPI}\|] & \text { NPI semantics } \\
\text { ii. } & \forall \mathrm{Q}^{\prime}\left[\mathrm{Q}^{\prime} \in|\ldots \mathrm{NPI} \ldots ?| \rightarrow\|\ldots \mathrm{NPI} \ldots ?\|>\mathrm{Q}^{\prime}\right] & \text { NPI condition }
\end{array}
$$

This leaves open the crucial question of how the ordering $>$ is to be defined. Naturally, the answer is dependent on assumptions about the semantics of questions, which is the topic of the next subsection.

\subsection{Question meanings and alternatives}

The QAL analysis being laid out here rests on the uncontroversial assumption that the semantics of a polar question determines a set of two propositions, viz. the proposition denoted by the question's prejacent and its negation. The polar question's denotation can be equated with that same set. Point-wise composition accordingly delivers an alternative set comprised of two-membered sets of propositions. For example, in (12), which repeats (1a), the prejacent is Ann eat anything, and so, with the abbreviation in (13), the denotation of (12) and its alternative set are as shown in 
(14a) and (14b), respectively.

(12) Did Ann eat anything?

(13) $\quad \|$ Ann eat anything $\|=\mathbf{a}$

a. $\quad \|$ did Ann eat anything? $\|=\{\mathbf{a},-\mathbf{a}\}$

b. $\quad \mid$ did Ann eat anything? $\mid=\left\{\left\{\mathbf{a}^{\prime},-\mathbf{a}^{\prime}\right\}: \mathbf{a}^{\prime} \in \mid\right.$ Ann eat anything $\left.\mid\right\}$

A wh-question denotation, too, can be taken to denote a set of propositions. Under a strongly exhaustive question meaning in the sense of Groenendijk \& Stokhof 1984, the members of a wh-question denotation (like the two members of a polar question denotation) partition the logical space. The members of this partition can be defined in terms of the wh-question's Hamblin answers. The Hamblin answers are the propositions obtained by applying the property given by the wh-phrase's scope to the elements in the extension of the wh-phrase's restrictor. In the wh-questions of interest here, the elements of the question partition can then be formed by conjoining those Hamblin answers or their negations. For example, in (15), which repeats (1b), the wh-phrase's restrictor is (of the) children, and its scope is ate anything. Assuming for purposes of illustration that the restrictor's extension is a set of just two individuals, the children a and $b$, and employing the abbreviation in (16), (15) has the Hamblin answers $\mathbf{A}(\mathrm{a})$ and $\mathbf{A}(\mathrm{b})$. The denotation of (15) is then the partition in (17a) and its alternative set is the set of partitions in (17b).

Which of the children ate anything?

$\|$ ate anything $\|=\mathbf{A}$

a. $\quad \|$ which of the children [ate anything]? $\|=$

$\{\mathbf{A}(\mathrm{a}) \cap \mathbf{A}(\mathrm{b}),-\mathbf{A}(\mathrm{a}) \cap \mathbf{A}(\mathrm{b}), \mathbf{A}(\mathrm{a}) \cap-\mathbf{A}(\mathrm{b}),-\mathbf{A}(\mathrm{a}) \cap-\mathbf{A}(\mathrm{b})\}$

b. $\quad \mid$ which of the children [ate anything]? $\mid=$

$\left\{\left\{\mathbf{A}^{\prime}(\mathrm{a}) \cap \mathbf{A}^{\prime}(\mathrm{b}),-\mathbf{A}^{\prime}(\mathrm{a}) \cap \mathbf{A}^{\prime}(\mathrm{b}), \mathbf{A}^{\prime}(\mathrm{a}) \cap-\mathbf{A}^{\prime}(\mathrm{b}),-\mathbf{A}^{\prime}(\mathrm{a}) \cap-\mathbf{A}^{\prime}(\mathrm{b})\right\}:\right.$ $\mathbf{A}^{\prime} \in \mid$ ate anything $\left.\mid\right\}$

Under the proposed semantics for polar and wh-questions, then, questions of both types denote sets of propositions that partition the logical space. As detailed below, van Rooy (2003) employs such a question semantics to define an ordering on question meanings intended to feed the theory of NPI licensing.

\subsection{Ordering questions}

Building on Krifka 1995, van Rooy 2003 proposes an ordering relation between questions in terms of questions' information theoretic entropy (Shannon 1948; Cover \& Thomas 2012). As stated in (18), for any question partitions Q and Q', Q is taken 
Questions as licensers

to be ordered above Q' just in case Q has greater entropy than Q', where the entropy of any partition $\mathrm{Q}$ is defined as in (19).

$$
\begin{aligned}
& \mathrm{Q}^{\prime} \mathrm{Q}^{\prime}: \Leftrightarrow \operatorname{Ent}_{\mathrm{Pr}_{\mathrm{s}}}(\mathrm{Q})>\operatorname{Ent}_{\mathrm{Pr}_{\mathrm{s}}}\left(\mathrm{Q}^{\prime}\right) \\
& \operatorname{Ent}_{\operatorname{Pr}}(\mathrm{Q}):=\sum_{\mathrm{q} \in \mathrm{Q}} \operatorname{Pr}(\mathrm{q}) \times \log _{2}\left(\frac{1}{\operatorname{Pr}(\mathrm{q})}\right)
\end{aligned}
$$

In (19), the entropy of a set of propositions $Q$ is defined relative to a probability mass function $\operatorname{Pr}$ with domain $\mathrm{Q}$, that is, a function that maps each member of $\mathrm{Q}$ to a probability such that the probabilities in the range of Pr sum up to $1 .^{3}$ In (18), the subscript $\mathrm{s}$ in $\operatorname{Pr}_{\mathrm{s}}$ indicates that the ordering of questions is intended to be relative to a probability mass function determined by the speaker's information state. The entropy of a question partition Q relative to any given probability mass function $\mathrm{Pr}$ is a measure of how evenly Pr distributes the probability mass over the propositions in $\mathrm{Q}$. Accordingly, the entropy of $\mathrm{Q}$ relative to $\mathrm{Pr}_{\mathrm{S}}$ is a measure of the speaker's uncertainty about which member of $\mathrm{Q}$ is true.

To illustrate, consider the question partitions Q1 and Q2 defined in (20). Table 1 specifies the entropy of these questions relative to a few selected probabilities mass functions.

$$
\begin{array}{ll}
\text { a. } & \mathrm{Q} 1=\{\mathrm{p},-\mathrm{p}\} \\
\text { b. } & \mathrm{Q} 2=\{\mathrm{p} \cap \mathrm{q},-\mathrm{p} \cap \mathrm{q}, \mathrm{p} \cap-\mathrm{q},-\mathrm{p} \cap-\mathrm{q}\}
\end{array}
$$

Table 1 illustrates that the entropy of a question is 0 if all the probability mass is in one of the cells of the partition (first and fifth row), and that it is maximal if all the cells of the partition have equal probability (third row). For a probability mass function determined by the speaker's information state, the former case amounts to the speaker's information state entailing a complete answer to the question, while the latter case amounts to the speaker being maximally uncertainty or unbiased as to the question's true answer. Probability mass functions that are between those two extremes determine entropy values that fall between 0 and the maximal entropy value (second and fourth row).

We will in the following be interested in how the entropy of a polar question denotation depends on the probability of the positive answer ( $p$ in Q1), and how the entropy of a wh-question denotation depends on the probabilities of the Hamblin answers ( $\mathrm{p}$ and $\mathrm{q}$ in Q2). For Q1 and Q2, these dependencies are plotted in Figure 1, where the right-hand curve assumes for Q2 that the Hamblin answers $p$ and $q$ are independent and have equal probability.

3 Note that this latter condition is necessarily met if Q partitions the logical space, as in the question meanings seen so far. However, we will later focus on cases where this condition is proposed to have a non-trivial effect, viz. cases of NPIs in phrasal disjunctive questions. 


\begin{tabular}{|c|c|c|c|c|c|c|c|}
\hline $\operatorname{Pr}(\mathrm{p})$ & $\operatorname{Pr}(-\mathrm{p})$ & Ent $_{\operatorname{Pr}}(\mathrm{Q} 1)$ & $\operatorname{Pr}(\mathrm{p} \cap \mathrm{q})$ & $\operatorname{Pr}(-\mathrm{p} \cap \mathrm{q})$ & $\operatorname{Pr}(p \cap-q)$ & $\operatorname{Pr}(-p \cap-q)$ & $\operatorname{Ent}_{\operatorname{Pr}}(\mathrm{Q} 2)$ \\
\hline 0 & 1 & 0 & 0 & 0 & 0 & 1 & 0 \\
\hline$\frac{1}{4}$ & $\frac{3}{4}$ & 0.81 & $\frac{1}{16}$ & $\frac{3}{16}$ & $\frac{3}{16}$ & $\frac{9}{16}$ & 1.62 \\
\hline$\frac{1}{2}$ & $\frac{1}{2}$ & 1 & $\frac{1}{4}$ & $\frac{1}{4}$ & $\frac{1}{4}$ & $\frac{1}{4}$ & 2 \\
\hline$\frac{3}{4}$ & $\frac{1}{4}$ & 0.81 & $\frac{9}{16}$ & $\frac{3}{16}$ & $\frac{3}{16}$ & $\frac{1}{16}$ & 1.62 \\
\hline 1 & 0 & 0 & 1 & 0 & 0 & 0 & 0 \\
\hline
\end{tabular}

Table 1
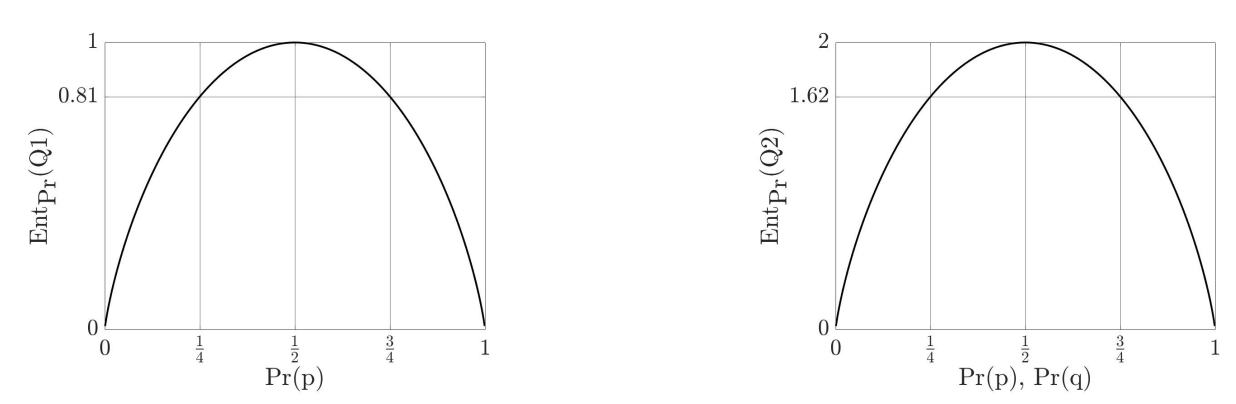

Figure 1

With an ordering of question denotations defined, we can now turn to applying this ordering in the analysis of weak NPIs in questions.

\subsection{Questions as licensers}

In (21), the rendition of the NPI theory for questions in (11) is updated by spelling out the ordering between questions appealed to in the NPI condition in (ii), in accordance with the definition in (18).

$$
\begin{array}{lll}
\text { i. } & \forall \mathrm{f}[\mathrm{f} \in|\mathrm{NPI}| \rightarrow \mathrm{f} \subset\|\mathrm{NPI}\|] & \text { NPI semantics } \\
\text { ii. } & \forall \mathrm{Q}^{\prime}\left[\mathrm{Q}^{\prime} \in|\ldots \mathrm{NPI} \ldots ?| \rightarrow\right. & \\
& \left.\operatorname{Ent}_{\mathrm{Pr}_{\mathrm{s}}}(\|\ldots \mathrm{NPI} \ldots ?\|)>\text { Ent }_{\mathrm{Pr}_{\mathrm{s}}}\left(\mathrm{Q}^{\prime}\right)\right] & \text { NPI condition }
\end{array}
$$

An essential and obvious desideratum is that the NPI condition be satisfiable for basic cases like the polar question in (12) and the wh-question in (15), repeated again in (22).

a. Did Ann eat anything?

b. Which of the children ate anything? 
Questions as licensers

This requirement is indeed met. For (22a), note first that the question semantics in (14) above guarantees (23a), that is, any alternative $\mathbf{a}$ ' to the actual positive answer $\mathbf{a}$ is strictly stronger than a itself. Given this, probability theory guarantees (23b), that is, no alternative positive answer $\mathbf{a}$ ' is more probable than the actual positive answer a.

$$
\begin{array}{ll}
\text { a. } & \forall \mathbf{a}^{\prime}\left[\mathbf{a}^{\prime} \in \mid \text { Ann eat anything } \mid \rightarrow \mathbf{a} \subset \mathbf{a}\right] \\
\text { b. } \quad \forall \mathbf{a}^{\prime}\left[\mathbf{a}^{\prime} \in \mid \text { Ann eat anything } \mid\right. & \left.\rightarrow \operatorname{Pr}(\mathbf{a}) \geq \operatorname{Pr}\left(\mathbf{a}^{\prime}\right)\right]
\end{array}
$$

Now suppose that $\operatorname{Pr}_{\mathrm{s}}(\mathbf{a})$, the probability of a given by the speaker's information state, is $\frac{1}{2}$ or less. Given (23), it can then be read off the left-hand curve in Figure 1 that the entropy of any alternative question is no greater than the entropy of the actual question; so, as long the probability of any alternative positive answer is different from the probability of the actual positive answer, the entropy of any alternative question will be strictly less than the entropy of the actual question. This entails that the NPI condition in (21) is satisfiable for polar questions, capturing the acceptability of examples like (22a).

With suitable adjustments, this assessment carries over to wh-questions like (22b). Suppose again that the wh-restrictor's extension is comprised of the two children a and $b$, and hence that the question semantics is as in (17) above. This semantics guarantees the truth of (24a), that is, any two alternative Hamblin answers $\mathbf{A}^{\prime}(\mathrm{a})$ and $\mathbf{A}^{\prime}(\mathrm{b})$ are strictly stronger than the actual Hamblin answers $\mathbf{A}(\mathrm{a})$ and $\mathbf{A}(\mathrm{b})$, respectively. Probability theory in turn guarantees the truth of $(24 b)$, that is, the probabilities of any two alternative Hamblin answers $\mathbf{A}^{\prime}(\mathrm{a})$ and $\mathbf{A}^{\prime}(\mathrm{b})$ are no greater than those of the actual Hamblin answers $\mathbf{A}(\mathrm{a})$ and $\mathbf{A}(\mathrm{b})$, respectively.

$$
\begin{gathered}
\text { a. } \quad \forall \mathbf{A}^{\prime}\left[\mathbf{A}^{\prime} \in \mid \text { ate anything } \mid \rightarrow \mathbf{A}^{\prime}(\mathrm{a}) \subset \mathbf{A}(\mathrm{a}) \& \mathbf{A}^{\prime}(\mathrm{b}) \subset \mathbf{A}(\mathrm{b})\right] \\
\text { b. } \quad \forall \mathbf{A}^{\prime}\left[\mathbf{A}^{\prime} \in \mid \text { ate anything } \mid \rightarrow\right. \\
\left.\operatorname{Pr}(\mathbf{A}(\mathrm{a})) \geq \operatorname{Pr}\left(\mathbf{A}^{\prime}(\mathrm{a})\right) \& \operatorname{Pr}(\mathbf{A}(\mathrm{b})) \geq \operatorname{Pr}\left(\mathbf{A}^{\prime}(\mathrm{b})\right)\right]
\end{gathered}
$$

Suppose now that $\operatorname{Pr}_{\mathrm{s}}(\mathbf{A}(\mathrm{a}))$ and $\operatorname{Pr}_{\mathrm{s}}(\mathbf{A}(\mathrm{b}))$ are equal and are both $\frac{1}{2}$ or less but not 0. Given (24), since the two Hamblin answers $\mathbf{A}(\mathrm{a})$ and $\mathbf{A}(\mathrm{b})$ are independent, we can then read off the right-hand curve in Figure 1 that any alternative question will have no greater entropy than the actual question; so, as long as the probabilities of any alternative Hamblin answers $\mathbf{A}^{\prime}$ (a) and $\mathbf{A}^{\prime}(b)$ are different from the probabilities of $\mathbf{A}(\mathrm{a})$ and $\mathbf{A}(\mathrm{b})$, respectively, the entropy of any alternative question will be strictly less than the entropy of the actual question. Hence, for the case of wh-questions too, the NPI condition in (21) is satisfiable, capturing the acceptability of examples like (22b).

With a rendition of van Rooy's (2003) entropy-based QAL analysis in place, we now are at last ready to return to the puzzle of NPIs in disjunctive questions 
introduced in section 1 .

\section{The disjunctive question puzzle solved}

To recap, the puzzle to be solved is the generalization that polar but not phrasal disjunctive questions license NPIs. This generalization is illustrated by the contrast in (3), which is repeated here as (25).

a. Did Ann eat anything or not?

polar

b. \#Did ANN eat anything or BEN?

phrasal

We will see that under the QAL analysis introduced above, the contrast falls out under independently motivated assumptions about the semantics of polar and phrasal disjunctive questions.

\subsection{Polar disjunctive questions}

Applying the NPI theory to polar disjunctive questions requires one to take a stand on the semantics of those questions. One natural hypothesis is that polar disjunctive questions have the same semantics as (non-disjunctive) polar questions. This view is natural enough to have been widely adopted in the literature, including, for example, Karttunen 1977, van Rooy \& Safarova 2003, and Guerzoni \& Sharvit 2014. Its benefits include the prediction that the two types of questions make identical contributions to truth conditions in cases where they appear embedded under a predicate like know, a prediction whose correctness is illustrated by the perceived truth conditional equivalence of (26a) and (26b). ${ }^{4}$

a. Ben knows [whether Ann ate something].

b. Ben knows [whether Ann ate something or not].

I will therefore adopt the view that disjunctive and non-disjunctive polar questions have the same semantics, and so I consider the two questions in (27), which once again reprints (3a) and (22a), to be semantically equivalent. Under current assumptions, then, (27a) has the denotation and alternative set given in (28).

4 Bolinger (1978) observed that polar questions and their polar disjunctive counterparts are not always interchangeable. For example, Bolinger reports that polar questions, but not polar disjunctive questions, can be used to extend an invitation. There is disagreement in the literature about what such contrasts entail about the semantic relation between the two types of questions. Biezma \& Rawlins (2012) propose different denotations for polar questions and their polar disjunctive counterparts. In contrast, van Rooy \& Safarova (2003) maintain that the two types of questions are semantically alike and that their differences can be understood in terms of pragmatics alone. The arguments made in this paper lead me to side with van Rooy \& Safarova. 
Questions as licensers

a. Did Ann eat anything or not?

b. Did Ann eat anything?

a. $\|$ did Ann eat anything or not? $\|=\{\mathbf{a},-\mathbf{a}\}$

b. $\quad \mid$ did Ann eat anything or not? $\mid=\left\{\left\{\mathbf{a}^{\prime},-\mathbf{a}^{\prime}\right\}: \mathbf{a}^{\prime} \in \mid\right.$ Ann eat anything $\left.\mid\right\}$

The QAL analysis of NPI licensing in questions outlined above then immediately predicts NPI licensing in polar alternative questions like (27a). After all, the theory only makes reference to a question's semantics, not to the syntactic structure that gives rise to this semantics. The analysis does not see the syntactic differences between disjunctive and non-disjunctive polar questions, predicting that the two behave alike in terms of NPI licensing. Therefore, since we have seen that the analysis correctly allows for NPI licensing by polar disjunctive questions, the same is guaranteed to hold for polar disjunctive questions. The first part of the generalization about NPIs in disjunctive questions has found a straightforward account.

\subsection{Phrasal disjunctive questions}

The second part of the generalization to be explained here is that, in contrast to polar disjunctive questions, NPIs are not licensed in phrasal disjunctive questions. To understand what might interfere with NPI licensing in phrasal disjunctive questions, it will be useful to compare them with wh-questions. What is the source of the contrast in (29) (which once again repeats (1b) and (3b))?

a. \#Did ANN eat anything or BEN?

b. Which of the children ate anything?

In an analysis that may suggest itself (e.g., Karttunen 1977; Krifka 2011), the disjoined propositions given by a phrasal disjunctive question, such as $\mathbf{A}(\mathrm{a})$ and $\mathbf{A}(\mathrm{b})$ in (29a), enter the denotation of such a question in the same way as Hamblin answers enter the denotation of a wh-question. Under present assumptions, this analysis leads to the semantics for (29a) given in (30).

a. $\quad$ did ANN eat anything or BEN? $\|=$ $\{\mathbf{A}(\mathrm{a}) \cap \mathbf{A}(\mathrm{b}),-\mathbf{A}(\mathrm{a}) \cap \mathbf{A}(\mathrm{b}), \mathbf{A}(\mathrm{a}) \cap-\mathbf{A}(\mathrm{b}),-\mathbf{A}(\mathrm{a}) \cap-\mathbf{A}(\mathrm{b})\}$

b. $\quad \mid$ did $A N N$ eat anything or BEN? $\mid=$ $\left\{\left\{\mathbf{A}^{\prime}(\mathrm{a}) \cap \mathbf{A}^{\prime}(\mathrm{b}),-\mathbf{A}^{\prime}(\mathrm{a}) \cap \mathbf{A}^{\prime}(\mathrm{b}), \mathbf{A}^{\prime}(\mathrm{a}) \cap-\mathbf{A}^{\prime}(\mathrm{b}),-\mathbf{A}^{\prime}(\mathrm{a}) \cap-\mathbf{A}^{\prime}(\mathrm{b})\right\}:\right.$ $\mathbf{A}^{\prime} \in \mid$ eat anything $\left.\mid\right\}$

Of course, this is the very semantics assigned above, in (17), to the wh-question (29b) under the assumption that the children are a and b. As noted, however, the QAL analysis introduced in section 3 predicts that semantically equivalent questions 
behave alike with regard to NPI licensing. Since, as seen, wh-questions are predicted to license NPIs, the same would have to be true for phrasal disjunctive questions. As matters stand, then, the second part of the generalization presents a challenge to this QAL analysis, rather the argument in its support advertised at the outset.

However, there are well-known observations about phrasal disjunctive questions that, independently from NPI licensing data, motivate a revision of the semantics in (30). Below I turn to these observations and the revisions they motivate.

\subsubsection{Presupposition of existence and uniqueness}

Phrasal disjunctive questions have been observed to carry an existence and uniqueness presupposition, the presupposition that exactly one of the two disjoined propositions is true (e.g. Karttunen \& Peters 1976; Bartels 1999; Biezma \& Rawlins 2012). As Biezma \& Rawlins (2012) observe, this presupposition can be detected, for example, by considering potential partial answers to a phrasal disjunctive question that merely state one of the hypothesized presuppositions. In (31), the intuited infelicity of the answers $\mathrm{B}$ and $\mathrm{C}$ can be taken to indicate that $\mathrm{B}$ and $\mathrm{C}$ do not provide even a partial answer to $\mathrm{A}$, as they are already entailed by what A presupposes - the existence presupposition in $\mathrm{B}$, and the uniqueness presupposition in $\mathrm{C}$.
A: Did ANN eat something or BEN?
B: \#At least one of the two did.
C: \#At most one of the two did.

The existence and uniqueness presuppositions can be encoded in the denotation of a phrasal disjunctive question by expunging from it those answers that are incompatible with those presuppositions. The semantic assumptions in (30) are then replaced with those in (32), where the propositions $\mathbf{A}(a) \cap \mathbf{A}(b)$ and $-\mathbf{A}(a) \cap-\mathbf{A}(b)$ are omitted from the question denotation, and likewise for the corresponding propositions in the alternative set. ${ }^{5}$

$$
\begin{array}{ll}
\text { a. } & \| \text { did } \mathrm{ANN} \text { eat anything or BEN? } \|= \\
& \{-\mathbf{A}(\mathrm{a}) \cap \mathbf{A}(\mathrm{b}), \mathbf{A}(\mathrm{a}) \cap-\mathbf{A}(\mathrm{b})\} \\
\text { b. } & \mid \text { did } \mathrm{ANN} \text { eat anything or BEN? } \mid= \\
& \left\{\left\{-\mathbf{A}^{\prime}(\mathrm{a}) \cap \mathbf{A}^{\prime}(\mathrm{b}), \mathbf{A}^{\prime}(\mathrm{a}) \cap-\mathbf{A}^{\prime}(\mathrm{b})\right\}: \mathbf{A}^{\prime} \in \mid \text { ate anything } \mid\right\}
\end{array}
$$

The existence plus uniqueness presupposition has thereby been encoded in the

5 This analysis effectively applies to phrasal disjunctive questions Higginbotham's (1993) proposal about how to semantically encode the presupposition of existence and uniqueness carried by singular which-questions. See Schwarz 2017 for NPI licensing in singular which-questions, which space reasons prevent me from discussing here. 
Questions as licensers

question meaning, as it amounts to the disjunction of the remaining propositions in the question denotation, i.e., $-\mathbf{A}(\mathrm{a}) \cap \mathbf{A}(\mathrm{b}) \cup \mathbf{A}(\mathrm{a}) \cap-\mathbf{A}(\mathrm{b})$.

Given this question semantics, what does the QAL analysis under consideration predict about NPI licensing? We will see below that this semantics renders the NPI condition impossible to satisfy, correctly capturing the generalization that NPIs are not licensed by phrasal disjunctive questions.

\subsubsection{The NPI condition cannot be met}

The following establishes that, given the NPI semantics in (21), reprinted in (33) below, and the presupposition posited for phrasal disjunctive questions, and assuming that the domain D is non-empty, the NPI condition as applied to such questions is impossible to meet: in these cases, it is impossible for the entropy of the actual question to differ from the entropies of all (in fact, any) of the alternatives.

$$
\begin{array}{lll}
\text { i. } & \forall \mathrm{f}[\mathrm{f} \in|\mathrm{NPI}| \rightarrow \mathrm{f} \subset\|\mathrm{NPI}\|] & \text { NPI semantics } \\
\text { ii. } & \forall \mathrm{Q}^{\prime}\left[\mathrm{Q}^{\prime} \in|\ldots \mathrm{NPI} \ldots ?| \rightarrow\right. & \\
& \left.\operatorname{Ent}_{\mathrm{Pr}_{\mathrm{s}}}(\|\ldots \mathrm{NPI} \ldots ?\|)>\text { Ent }_{\mathrm{Pr}_{\mathrm{s}}}\left(\mathrm{Q}^{\prime}\right)\right] & \text { NPI condition }
\end{array}
$$

To begin, by entailing that no alternative question has the same entropy as the actual question, the NPI condition entails that there is no alternative question whose Hamblin answers have the same probabilities as the corresponding actual Hamblin answers:

$$
\begin{gathered}
\text { [1] } \neg \exists \mathbf{A}^{\prime}\left[\mathbf{A}^{\prime} \in \mid \text { eat anything } \mid \& \operatorname{Pr}_{\mathrm{s}}(\mathbf{A}(\mathrm{a}))=\operatorname{Pr}_{\mathrm{s}}\left(\mathbf{A}^{\prime}(\mathrm{a})\right) \&\right. \\
\left.\operatorname{Pr}_{\mathrm{s}}(\mathbf{A}(\mathbf{b}))=\operatorname{Pr}_{\mathrm{s}}\left(\mathbf{A}^{\prime}(\mathrm{b})\right)\right]
\end{gathered}
$$

However, as shown below, given the existence and uniqueness presupposition, and given the NPI semantics, the NPI condition also implies the negation of [1].

As noted earlier, for the entropy of a set of propositions to be defined relative to a probability mass function, the probabilities assigned to the members of that set must sum to 1 . So the NPI condition imposes the requirement that for the actual question, the answers' probabilities given by the speaker's epistemic state sum to 1 . Given that the domain $\mathrm{D}$, and hence the alternative set for ate anything, is non-empty by assumption, the same requirement holds for some of the alternative questions (in fact, it holds for all alternatives):

$$
\begin{array}{cc}
\text { [2] a. } & \operatorname{Pr}_{\mathrm{s}}(-\mathbf{A}(\mathrm{a}) \cap \mathbf{A}(\mathrm{b}))+\operatorname{Pr}_{\mathrm{s}}(\mathbf{A}(\mathrm{a}) \cap-\mathbf{A}(\mathrm{b}))=1 \\
\text { b. } & \exists \mathbf{A}^{\prime}\left[\mathbf{A}^{\prime} \in \mid \text { eat anything } \mid \& \operatorname{Pr}_{\mathrm{s}}\left(-\mathbf{A}^{\prime}(\mathrm{a}) \cap \mathbf{A}^{\prime}(\mathrm{b})\right)+\right. \\
& \left.\operatorname{Pr}_{\mathrm{s}}\left(\mathbf{A}^{\prime}(\mathrm{a}) \cap-\mathbf{A}^{\prime}(\mathrm{b})\right)=1\right]
\end{array}
$$

Probability theory then ensures that the probabilities of the Hamblin answers to 
the actual question must sum to 1, and likewise for the probabilities of the Hamblin answers to some (in fact, all) of the alternative questions: ${ }^{6}$

$$
\begin{array}{ll}
\text { [3] a. } & \operatorname{Pr}_{\mathrm{s}}(\mathbf{A}(\mathrm{a}))+\operatorname{Pr}_{\mathrm{s}}(\mathbf{A}(\mathrm{b}))=1 \\
\text { b. } & \exists \mathbf{A}^{\prime}\left[\mathbf{A}^{\prime} \in \mid \text { eat anything } \mid \& \operatorname{Pr}_{\mathrm{s}}\left(\mathbf{A}^{\prime}(\mathrm{a})\right)+\operatorname{Pr}_{\mathrm{s}}\left(\mathbf{A}^{\prime}(\mathrm{b})\right)=1\right]
\end{array}
$$

However, we have already observed (as stated in (24) above) that, due to the NPI semantics, a Hamblin answer to any of the alternative questions cannot be more probable than the corresponding Hamblin answer to the actual question:

[4] $\forall \mathbf{A}^{\prime}\left[\mathbf{A}^{\prime} \in \mid\right.$ eat anything $\mid \rightarrow \operatorname{Pr}_{\mathrm{s}}(\mathbf{A}(\mathrm{a})) \geq \operatorname{Pr}_{\mathrm{s}}\left(\mathbf{A}^{\prime}(\mathrm{a})\right) \&$

$$
\left.\operatorname{Pr}_{\mathrm{s}}(\mathbf{A}(\mathrm{b})) \geq \operatorname{Pr}_{\mathrm{s}}\left(\mathbf{A}^{\prime}(\mathrm{b})\right)\right]
$$

And so, since the probabilities of the Hamblin answers must sum to 1 in the actual question (as per [3a]) and some alternative (as per [3b]), it follows that the Hamblin answers in that alternative must have the same probabilities as the corresponding actual Hamblin answers:

$$
\begin{gathered}
\text { [5] } \exists \mathbf{A}^{\prime}\left[\mathbf{A}^{\prime} \in \mid \text { eat anything } \mid \& \operatorname{Pr}_{\mathrm{s}}(\mathbf{A}(\mathrm{a}))=\operatorname{Pr}_{\mathrm{s}}\left(\mathbf{A}^{\prime}(\mathrm{a})\right) \&\right. \\
\left.\operatorname{Pr}_{\mathrm{s}}(\mathbf{A}(\mathrm{b}))=\operatorname{Pr}_{\mathrm{s}}\left(\mathbf{A}^{\prime}(\mathrm{b})\right)\right]
\end{gathered}
$$

Which entails that there is some alternative question that has the same entropy as the actual question, contradicting the requirement, stated in [1], that there be no such alternative.

In sum, for phrasal disjunctive questions, the NPI condition is not satisfiable, and the finding that such questions do not license NPIs has be given a principled account.

\section{A complication: mere existence presuppositions}

However, this account requires a refinement. For reasons detailed below, the NPI condition turns out to be too strong to do justice to the full range of data.

The NPI licensing contrast between the polar disjunctive question in (29a) and the wh-question (29b), repeated below as (34), has here been credited to the presupposition of existence and uniqueness attested in the former. However, according to a prevalent intuition, wh-questions, too, carry a presupposition, viz. a presupposition of existence (e.g., Karttunen 1977). For (29b), this is the presupposition that at least one of the children ate something.

$$
\text { Which of the children ate anything? }
$$

6 Sketch of proof: let $\operatorname{Pr}(-\mathrm{p} \cap \mathrm{q})+\operatorname{Pr}(\mathrm{p} \cap-\mathrm{q})=1$; this guarantees $\operatorname{Pr}(\mathrm{p} \cap \mathrm{q})=0$, hence both $\operatorname{Pr}(-\mathrm{p} \cap$ $\mathrm{q})+\operatorname{Pr}(\mathrm{p} \cap-\mathrm{q})=\operatorname{Pr}(\mathrm{p} \cup \mathrm{q})$ and $\operatorname{Pr}(\mathrm{p})+\operatorname{Pr}(\mathrm{q})=\operatorname{Pr}(\mathrm{p} \cup \mathrm{q})$, and therefore $\operatorname{Pr}(\mathrm{p})+\operatorname{Pr}(\mathrm{q})=1$. 
Questions as licensers

\begin{tabular}{c|c|c|c}
$\operatorname{Pr}(\mathrm{p} \cap \mathrm{q})$ & $\operatorname{Pr}(-\mathrm{p} \cap \mathrm{q})$ & $\operatorname{Pr}(\mathrm{p} \cap-\mathrm{q})$ & $\operatorname{Ent}_{\mathrm{Pr}}(\mathrm{Q} 3)$ \\
\hline 0 & $\frac{1}{2}$ & $\frac{1}{2}$ & 1 \\
$\frac{1}{3}$ & $\frac{1}{3}$ & $\frac{1}{3}$ & 1.59 \\
1 & 0 & 0 & 0
\end{tabular}

Table 2

In analogy to the above treatment of phrasal disjunctive questions, the existence presupposition of a wh-question is to be entered in the question denotation by expunging from it the proposition that is inconsistent with the presupposition, and likewise for the alternative sets of propositions. Again supposing for illustration that the wh-restrictor extension is comprised of the two children a and b, this amounts to replacing (17) above with (35) below.

a. $\quad$ which of the children ate anything? $\|=$ $\{-\mathbf{A}(\mathrm{a}) \cap \mathbf{A}(\mathrm{b}), \mathbf{A}(\mathrm{a}) \cap-\mathbf{A}(\mathrm{b}), \mathbf{A}(\mathrm{a}) \cap \mathbf{A}(\mathrm{b})\}$

b. $\quad \mid$ which of the children ate anything? $\mid=$ $\left\{\left\{-\mathbf{A}^{\prime}(\mathrm{a}) \cap \mathbf{A}^{\prime}(\mathrm{b}), \mathbf{A}^{\prime}(\mathrm{a}) \cap-\mathbf{A}^{\prime}(\mathrm{b}), \mathbf{A}^{\prime}(\mathrm{a}) \cap \mathbf{A}^{\prime}(\mathrm{b})\right\}: \mathbf{A}^{\prime} \in \mid\right.$ ate anything $\left.\mid\right\}$

Under this revised semantics, the question denotation again encodes an existence presupposition as the disjunction of the propositions it contains, which is now equivalent to $\mathbf{A}(\mathrm{a}) \cup \mathbf{A}(\mathrm{b})$.

How does the NPI condition in (33) apply to (29a) under the revised semantics in (35)? To address this question, consider the information given in Table 2 and Figure 2 about the set of propositions Q3 defined in (36) below. Table 2 shows the entropy of Q3 for selected probability mass functions with domain Q3. This table provides background for Figure 2, which, assuming that $\operatorname{Pr}(\mathrm{p})$ equals $\operatorname{Pr}(\mathrm{q})$, shows how the probability of $\mathrm{p}$ and $\mathrm{q}$ determines the entropy of Q3.

$$
\mathrm{Q} 3=\{-\mathrm{p} \cap \mathrm{q}, \mathrm{p} \cap-\mathrm{q}, \mathrm{p} \cap \mathrm{q}\}
$$

What stands out about Figure 2 is the limitation of the $\mathrm{x}$-axis to the interval from $\frac{1}{2}$ to 1 . The reason for this limitation is that, assuming $\operatorname{Pr}(\mathrm{p})$ equals $\operatorname{Pr}(\mathrm{q})$, probability theory requires that any probability mass function from Q3 map p and $q$ to a probability of no less than $\frac{1}{2}$. So, relative to any probability function that maps $p$ and $q$ to a probability of less than $\frac{1}{2}$, the entropy of Q3 fails to be defined. Dropping the assumption that $\operatorname{Pr}(\mathrm{p})$ equals $\operatorname{Pr}(\mathrm{q})$, it holds more generally that any probability mass function from Q3 maps $\mathrm{p}$ and $\mathrm{q}$ to probabilities that sum to at least 1, and hence that the entropy of Q3 is undefined relative to any probability function that does not 


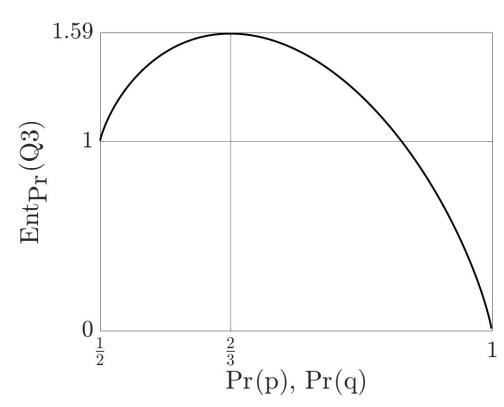

Figure 2

meet this constraint. ${ }^{7}$ More generally still, probability theory can be shown to ensure that a question denotation that encodes an existence presupposition, whatever the number of Hamblin answers it might be based on, will not have a defined entropy unless the probabilities of those Hamblin answers sum to at least 1.

To appreciate the significance of this fact for (34) under the semantics in (35), recall from section 2 the observation that the alternative set for ate anything, if non-empty, includes among its members the impossible property, the property that maps any individual in its domain to the contradictory proposition. For this choice of A', then, all the Hamblin answers become equivalent by collapsing into the contradictory proposition. To be sure, that alternative Hamblin answer will not be mapped to 1 by the function $\operatorname{Pr}_{\mathrm{s}}$, the probability function determined by the speaker's information state. Which ensures that for the wh-question (34) under the semantics (35), there exists an alternative question whose entropy is undefined. As matters stand, therefore, even for the acceptable wh-question (34), the NPI condition as stated in (33) turns out to not be satisfiable.

This suggests that the NPI condition for questions in (21) above is too strong. The condition should not be construed a requiring that every alternative to the actual question have an entropy that is lower than the entropy of the actual question. To capture all the relevant data, the condition should instead require (i) that there be an alternative question whose entropy is defined and (ii) that for each alternative question whose entropy is defined, it is less than the entropy of the actual question. With this revision, the rendition of the NPI theory for questions in (21) above is to be replaced with (37) below.

7 Sketch of proof: the assumption $\operatorname{Pr}(-\mathrm{p} \cap \mathrm{q})+\operatorname{Pr}(\mathrm{p} \cap-\mathrm{q})+\operatorname{Pr}(\mathrm{p} \cap \mathrm{q})=1$ guarantees $\operatorname{Pr}(\mathrm{p} \cup \mathrm{q})=1$; given that $\operatorname{Pr}(\mathrm{p} \cup \mathrm{q}) \leq \operatorname{Pr}(\mathrm{p})+\operatorname{Pr}(\mathrm{q})$ (an instance of the so-called union bound) it follows that $\operatorname{Pr}(\mathrm{p})+\operatorname{Pr}(\mathrm{q}) \geq 1$. 
Questions as licensers

i. $\quad \forall \mathrm{f}[\mathrm{f} \in|\mathrm{NPI}| \rightarrow \mathrm{f} \subset\|\mathrm{NPI}\|]$

ii. $\exists Q^{\prime}\left[Q^{\prime} \in \mid \ldots\right.$ NPI ...? $\mid \& \operatorname{Ent}_{\operatorname{Pr}_{\mathrm{s}}}\left(Q^{\prime}\right)$ is defined $] \&$ $\forall Q^{\prime}\left[Q^{\prime} \in \mid \ldots\right.$ NPI $\ldots ? \mid \& \operatorname{Ent}_{\mathrm{Pr}_{\mathrm{s}}}\left(\mathrm{Q}^{\prime}\right)$ is defined $\rightarrow$

$\left.\operatorname{Ent}_{P_{\mathrm{s}}}(\|\ldots \mathrm{NPI} \ldots ?\|)>\operatorname{Ent}_{\mathrm{Pr}_{\mathrm{s}}}\left(\mathrm{Q}^{\prime}\right)\right]$
NPI semantics

NPI condition

As intended, the revised definition renders the NPI condition satisfiable for the question in (34) under the semantics in (35): as can be read off Figure 2, the NPI condition in (37) can be satisfied, in particular, if $\operatorname{Pr}_{\mathrm{s}}(\mathbf{A}(\mathrm{a}))$ and $\operatorname{Pr}_{\mathrm{s}}(\mathbf{A}(\mathrm{a}))$ are equal and are both $\frac{2}{3}$ or less but greater than $\frac{1}{2}$; in that case, for reasons familiar from the exposition in section 3, the entropy of any alternative question, if defined, cannot be greater than the entropy of the actual question, and will be strictly less than the actual question's entropy as long as the corresponding alternative Hamblin answers are assigned different probabilities than the actual Hamblin answers.

At the same time, under this revised NPI theory for questions, the existence plus uniqueness presupposition assumed for phrasal disjunctive questions continues to prevent the NPI condition from being satisfied. As can be read off the the proof presented in 4.2.2, a presupposition of existence and uniqueness forces the entropy of any alternative question, if defined, to be identical to the entropy of the actual question. The revision of the NPI condition proposed here therefore preserves the main result of this paper. Phrasal disjunctive question are still predicted to not license NPIs, on the grounds of that in such cases the NPI condition is not satisfiable.

\section{Conclusion}

The questions-are-licensers analysis based on question entropy (Krifka 1995; van Rooy 2003) offers a theoretically parsimonious solution to the puzzle of NPIs in disjunctive questions. In particular, it makes the welcome prediction that the existence plus uniqueness presupposition that is independently attested in phrasal disjunctive questions makes the licensing conditions for an NPI impossible to satisfy.

The questions-introduce-licensers analyses of Nicolae $(2013,2015)$ and Guerzoni \& Sharvit (2014) make different predictions, predictions that are in conflict with the generalization about disjunctive questions assumed in this paper. These authors considered those different predictions to be correct. However, to the extent that the generalization assumed here is accepted (see footnote 2), the findings in this paper constitute an argument for the questions-are-licensers account explored here over the competing questions-introduce-licensers analyses. 


\section{References}

Bartels, Christine. 1999. The Intonation of English Statements and Questions: A Compositional Interpretation. New York and London: Garland Publishing.

Biezma, María \& Kyle Rawlins. 2012. Responding to alternative and polar questions. Linguistics and Philosophy 361-406. doi:10.1007/s10988-012-9123-z.

Biezma, María \& Kyle Rawlins. 2015. Alternative questions. Language and Linguistics Compass 9(11). 450-468. doi:10.1111/lnc3.12161.

Bolinger, Dwight. 1978. Yes-No questions are not alternative questions. In Henry Hiż (ed.), Questions, 87-105. Dordrecht: Springer.

Chierchia, Gennaro. 2013. Logic in Grammar: Polarity, Free Choice, and Intervention. Oxford: Oxford University Press.

Cover, Thomas M \& Joy A Thomas. 2012. Elements of Information Theory. John Wiley \& Sons.

Groenendijk, Jeroen \& Martin Stokhof. 1984. Studies on the semantics of questions and the pragmatics of answers: University of Amsterdam PhD dissertation.

Guerzoni, Elena \& Yael Sharvit. 2014. "Whether or not anything" but not "whether anything or not". In Luka Crnič \& Uli Sauerland (eds.), The Art and Craft of Semantics: a Festschrift for Irene Heim, 199-224. Cambride, MA: MIT Working Papers in Linguistics (MITWPL).

Hamblin, Charles L. 1973. Questions in Montague English. Foundations of Language 10. 41-53.

Higginbotham, James. 1993. Interrogatives. In Kenneth Hale \& Jay Keyser (eds.), The View from Building 20, Cambride, MA: MIT Press.

Kadmon, Nirit \& Fred Landman. 1990. Polarity sensitive any and free choice any. In Martin Stokhof \& L. Toorenvliet (eds.), 7th Amsterdam Colloquium, 227-251. ITLT.

Kadmon, Nirit \& Fred Landman. 1993. Any. Linguistics and Philosophy 16. 353-422. doi:10.1007/BF00985272.

Karttunen, Lauri. 1977. Syntax and semantics of questions. Linguistics and Philosophy 1. 3-44. doi:10.1007/BF00351935.

Karttunen, Lauri \& Stanley Peters. 1976. What indirect questions conventionally implicate. In Salikoko S. Mufwene (ed.), 12th Regional Meeting of the Chicago Linguistic Society Chicago, 351-368. Chicago, Illinois.

Krifka, Manfred. 1995. The semantics and pragmatics of polarity items. Linguistic Analysis 25(3-4). 209-257.

Krifka, Manfred. 2003. Polarity items in questions. slides for presentation at Workshop on Polarity, Scalar Phenomena, Implicatures: At the Interface between Grammar and the Cognitive System. June 18-20, 2003, University MilanBicocca. http://amor.cms.hu-berlin.de/ h2816i3x/Talks/PolarityQuestions.ppt. 
Questions as licensers

Krifka, Manfred. 2011. Questions. In Klaus von Heusinger, Claudia Maienborn \& Paul Portner (eds.), Semantics - An International Handbook of Natural Language Meaning, 1742-1785. de Gruyter.

Ladusaw, William. 1979. Polarity sensitivity as inherent scope relations: University of Texas at Austin PhD dissertation.

Lahiri, Utpal. 1998. Focus and negative polarity in Hindi. Natural Language Semantics 6(1). 57-123. doi:10.1023/A:1008211808250.

Nicolae, Andreea C. 2013. Any questions? Polarity as a window into the structure of questions: Harvard University $\mathrm{PhD}$ dissertation.

Nicolae, Andreea C. 2015. Questions with NPIs. Natural Language Semantics 23(1). 21-76. doi:10.1007/s11050-014-9110-8.

Rooth, Mats. 1985. Association with focus: University of Massachusetts Amherst $\mathrm{PhD}$ dissertation.

van Rooy, Robert. 2003. Negative polarity items in questions: Strength as relevance. Journal of Semantics 20(3). 239-273.

van Rooy, Robert \& Marie Safarova. 2003. On polar questions. In R. Young \& Y. Zhou (eds.), Semantics and Linguistic Theory (SALT) 13, 292-309. Ithaca, NY: CLC Publications.

Schwarz, Bernhard. 2017. On the locus of question exhaustification. Talk at North East Linguistic Society (NELS) 48, University of Iceland.

Shannon, Claude Elwood. 1948. A mathematical theory of communication. Bell System Technical Journal 27. 379-423.

Bernhard Schwarz

Department of Linguistics

1085 Dr. Penfield

McGill University

Montreal, QC H3A 1A7

bernhard.schwarz@mcgill.ca 